\title{
Tensile behaviour of drawn tungsten wire used in tungsten fibre-reinforced tungsten composites
} reinforced tungsten composites

\section{Introduction}

Tungsten $(\mathrm{W})$ is the main candidate for highly loaded areas in a future fusion reactor due to its excellent erosion resistance and low $\mathrm{H}$ retention as well as high temperature

E-mail: johann.riesch@ipp.mpg.de

\begin{abstract}
In tungsten fibre-reinforced tungsten composites $\left(\mathrm{W}_{\mathrm{f}} / \mathrm{W}\right)$ the brittleness problem of tungsten is solved by utilizing extrinsic toughening mechanisms. The properties of the composite are very much related to the properties of the drawn tungsten wire used as fibre reinforcements. Their high strength and capability of ductile deformation are ideal properties facilitating toughening of $\mathrm{W}_{\mathrm{f}} / \mathrm{W}$. Tensile tests have been used for determining mechanical properties and study the deformation and the fracture behaviour of the wire. Tests of as-fabricated and straightened drawn wires with a diameter between $16 \mu \mathrm{m}$ and $150 \mu \mathrm{m}$ as well as wire electrochemically thinned to a diameter of $5 \mu \mathrm{m}$ have been performed. Engineering stress-strain curves and a microscopic analysis are presented with the focus on the ultimate strength. All fibres show a comparable stress-strain behaviour comprising necking followed by a ductile fracture. A reduction of the diameter by drawing leads to an increase of strength up to $4500 \mathrm{MPa}$ as a consequence of a grain boundary hardening mechanism. Heat treatment during straightening decreases the strength whereas electrochemical thinning has no significant impact on the mechanical behaviour.
\end{abstract}

Keywords: tungsten, drawn wire, strength, grain boundary hardening, tungsten fibrestrength and creep resistance combined with a high thermal conductivity and melting

\author{
J. Riesch ${ }^{1}$, A. Feichtmayer ${ }^{1,2}$, M. Fuhr ${ }^{1,3}$, J. Almanstötter ${ }^{4}$, \\ J.W. Coenen ${ }^{5}$, H. Gietl ${ }^{1,2}$, T. Höschen ${ }^{1}$, Ch. Linsmeier $^{5}$, R. \\ $\mathrm{Neu}^{1,2}$ \\ ${ }^{1}$ Max-Planck-Institut für Plasmaphysik, 85748 Garching, Germany \\ 2 Technische Universität München, 85748 Garching, Germany \\ ${ }^{3}$ Department of Materials Science, Glass and Ceramics, University of \\ Erlangen-Nürnberg, Martensstr. 5, Erlangen, Germany \\ ${ }^{4}$ OSRAM GmbH, Corporate Technology CT TSS MTS MET, 86830 \\ Schwabmünchen, Germany \\ ${ }^{5}$ Forschungszentrum Jülich GmbH, Institut für Energie- und Klimaforschung - \\ Plasmaphysik, 52425 Jülich, Germany
}


point [1]. However, $\mathrm{W}$ suffers from an intrinsic brittleness up to a temperature of typically $500 \mathrm{~K}-600 \mathrm{~K}$ [2] and is prone to operational embrittlement e.g. by grain coarsening [3] and/or neutron irradiation [4]. In tungsten fibre-reinforced tungsten composites $\left(\mathrm{W}_{\mathrm{f}} / \mathrm{W}\right)$ the brittleness problem is solved by utilizing extrinsic toughening mechanisms similar to those found in ceramic fibre-reinforced ceramic composites $[5,6,7]$. The properties of the composite are very much related to the properties of the $\mathrm{W}$ wire used as fibre reinforcement $[8,9]$. Their high strength and capability of ductile deformation are ideal properties facilitating toughening of $\mathrm{W}_{\mathrm{f}} / \mathrm{W}$. The high strength is important for the bridging effect and ductile deformation allows the dissipation of a substantial amount of energy [10, 11].

The major application of drawn $\mathrm{W}$ wire historically emerged from its use as filament material in incandescent lightning [12]. Here a key parameter is the creep resistance at high temperature which has been significantly improved by doping pure tungsten wire with small amounts of potassium. The main focus of the research on $\mathrm{W}$ wire has been laid for many years in the further development of the manufacturing process and high temperature stability. However, if used as reinforcements in composites such as $\mathrm{W}_{\mathrm{f}} / \mathrm{W}$ the performance at lower temperature in general as well as basic mechanical properties like strength or fracture behaviour are becoming more important.

In this context, both pure and potassium doped tungsten wire with a diameter of $150 \mu \mathrm{m}$ have been investigated in tensile tests at room temperature in the as-fabricated and annealed state $[13,8]$. Both wire types show comparable behaviour featuring high strength and ductility as long as the elongated fine grain structure is conserved. The loss of this structure by recrystallisation accompanied by massive grain growth leads to embrittlement. Tensile tests at temperatures up to $600{ }^{\circ} \mathrm{C}$ on identical wires revealed a good high temperature strength [14]. Beside the threshold temperature for embrittlement no significant difference in the behaviour of doped and undoped wire was observed. Recently the ductile behaviour has been matter of detailed investigation [15].

Already in 1913 W.D. Coolidge [16] discovered that the room temperature ductility of tungsten wire increases with increasing deformation. The high deformation state is a consequence of the typical manufacturing process. The first step in the production of tungsten wire is the preparation of the $\mathrm{W}$ powder and the possible doping with potassium. Powder pressing is used to form a green body which is then sintered by direct current to form an ingot. This ingot is bar rolled and swagged and finally drawn down to a diameter of 2 to $4 \mathrm{~mm}$. The wire is subsequently drawn through dies with decreasing size until the final diameter is reached. The drawing temperature starts at $1000^{\circ} \mathrm{C}$ and is decreased with decreasing diameter. The reduction in area per drawing step lies between $40 \%$ at the beginning and 10\% in the end. As the strength and hardness significantly increases during each drawing step intermediate annealing steps at a temperature of $1600{ }^{\circ} \mathrm{C}$ are necessary to prevent overworking. Since the number of drawing steps is proportional to the desired wire diameter, the total deformation increases with decreasing wire thickness. Details of the process can be found in [17].

In this contribution we present the results of tensile tests at room temperature of 
commercially available as-fabricated and straightened drawn and potassium doped W wire. Wire with diameters of $16 \mu \mathrm{m}, 50 \mu \mathrm{m}, 100 \mu \mathrm{m}$ and $150 \mu \mathrm{m}$ as well as $16 \mu \mathrm{m}$ wire electrochemically thinned to $5 \mu \mathrm{m}$ have been tested. Engineering stress-strain curves and microscopical observation of the fracture surface are presented. The aim is to compare the mechanical behaviour of the different wire types and provide strength values to support the understanding and future design of $\mathrm{W}_{\mathrm{f}} / \mathrm{W}$ composites. This study is necessary as existing work mainly focusses on its use as a lightning filament and mechanical data inevitable for composite development is not available in the needed precision and extend.

\section{Experimental}

As-fabricated and straightened drawn tungsten wires were provided by the OSRAM GmbH, Schwabmünchen. The wires were doped with 60-75 ppm potassium. The straightening was done by mechanical loading via stretching at elevated temperature covering stages IV and V of recovery in cold deformed tungsten [18] witch lie typically between 500 and $1000{ }^{\circ} \mathrm{C}$. The wires were delivered on spools and were cut by a tungsten carbide nipper or scissors (for the very thin wires) to a length of $80-90 \mathrm{~mm}$. These wire pieces are called fibres in the following.

Technical constraints do not allow a diameter reduction below $16 \mu \mathrm{m}$ by drawing. Electrochemical thinning was therefore used to reduce the diameter to approximately $5 \mu \mathrm{m}$. A $16 \mu \mathrm{m}$ fibre is coated by an isolating protective lacquer apart from a $2 \mathrm{~mm}$ long region in the centre. For the electro thinning this fibre is mounted electrically conductive onto a rotatable sample holder and put into an electrolyte (mixture of $\mathrm{NaOH}, \mathrm{H}_{2} \mathrm{O}$ and glycerine; details in [19]). A voltage of $7 \mathrm{~V}$ accompanied by a constant rotation leads to a uniform removal of material in the uncoated area until the target diameter is reached. It has to be noted that not soluble carbon probably remaining from the fabrication process, led to inhomogeneities in some cases. An overview of all tested wire types is given in table 1.

The tensile tests were performed with a universal testing machine (TIRA Test 2820) at room temperature. A $200 \mathrm{~N}$ range load cell was used for the fibres of the type 150 and 150-st (see table 1 for IDs) and a $20 \mathrm{~N}$ range load cell for thinner fibres. For the 150 type fibres the displacement was measured by the cross head displacement corrected by the machine stiffness. In the other cases a contactless optical measurement system was established. In this system the fibre is monitored by a ccd camera combined with a telecentric lens throughout the test. This lens displays an orthographic view and thus allows the geometrically correct tracking of characteristic features on the object. By tracking the movement of these points the displacement and thus the engineering strain is calculated using a LabView based program. As we use two systems for strain measurments we avoid quanitative statements on strain values in this article.

The fibre ends were embedded into a two component epoxy glue (UHU Plus endfest 300). The cross-section in the embedded area is enlarged and thus the probability of 


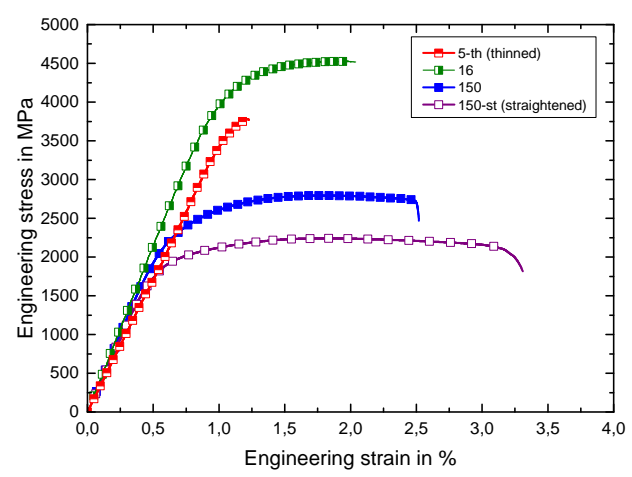

Figure 1. Typical engineering stress - strain curves for 5-th,16,150 and 150-st fibres. Please note that the measured data points are represented by the shown lines and the symbols are only used to make the curves distinguishable.

fracture in this area is reduced (compare procedure described in [8]). Except for the 150 type fibre the fibres were attached to a paper frame by the glue allowing an easy

handling. The sides of the frame parallel to the fibre were cut after mounting the sample into the testing device.

For the 150 type fibre (no paper frame) the measuring length was defined by the fibre length between the epoxy embedding which was between 25 to $30 \mathrm{~mm}$. In the case of the optical strain measurement the distance of the reference points on the fibre defines the measuring length. For 5-th fibres the transition points to the thinned centre parts were used as reference points. For all other fibre types the distance of $30 \mathrm{~mm}$ between the inner edges of the paper frame was used. The tensile test were performed in a displacement controlled mode with a constant cross-head speed (see table 1 for values).

The fracture surface of all samples was investigated by optical microscopy and for selected samples using a FEI Helios NanoLab 600 scanning electron microscope (SEM). As a measure for deformation, the reduced diameter was determined for selected fibre types using the SEM images.

\section{Results}

Typical engineering stress-strain curves for selected fibre types are shown in figure 1. All curves show ductile characteristics. After a region of elastic deformation, strain hardening is observed. For the straightened fibre the strain hardening is less pronounced. Then a plateau is observed in which the maximum load is reached. In this region the stress changes only moderately. For fibres with a diameter larger than $16 \mu \mathrm{m}$ a pronounced load drop occurs prior to final fracture. The plateau as well as this pronounced load drop was not detectable for the 5-th type fibre. Similar characteristics were also observed for fibres with a diameter of $50 \mu \mathrm{m}$ and $100 \mu \mathrm{m}$, however, relatively large variations in the elastic region maybe due to inaccuracies in the strain measurement were detected. Therefore, the ultimate strength as well as the reduction in cross-section 
Table 1. Overview of tested wire types and results for ultimate strength and reduction of diameter. The uncertainties for the strength are calculated as described in Appendix A. The uncertainties for the reduced area are calculated as standard deviation of the mean.

\begin{tabular}{ccccccc}
\hline ID & $\begin{array}{c}\text { Nominal } \\
\text { diameter } \\
\text { in } \mu \mathrm{m}\end{array}$ & $\begin{array}{c}\text { Cross-head } \\
\text { displacement } \\
\text { in } \mu \mathrm{m} \mathrm{s}^{-1}\end{array}$ & $\begin{array}{c}\text { Number of } \\
\text { valid tests }\end{array}$ & $\begin{array}{c}\text { Ultimate } \\
\text { strength } \\
\sigma_{\mathrm{u}} \text { in MPa }\end{array}$ & $\begin{array}{c}\text { Reduction } \\
\text { in area in } \\
\%\end{array}$ & $\begin{array}{c}\text { Preparation } \\
\text { state }\end{array}$ \\
\hline 5 -th & 5 & 0.5 & 3 & $4035 \pm 112$ & $55 \pm 2$ & thinned \\
16 & 16 & 1 & 10 & $4481 \pm 35$ & $49 \pm 1$ & as-fabricated \\
16 & 16 & 5 & 3 & $4550 \pm 67$ & - & as-fabricated \\
50 & 50 & 5 & 8 & $2935 \pm 27$ & - & as-fabricated \\
$50-s t$ & 50 & 5 & 8 & $2405 \pm 22$ & - & straightened \\
100 & 100 & 5 & 7 & $2152 \pm 17$ & - & as-fabricated \\
$100-s t$ & 100 & 5 & 7 & $2167 \pm 17$ & - & straightened \\
150 & 150 & 5 & 7 & $2774 \pm 29$ & $37 \pm 3$ & as-fabricated \\
$150-s t$ & 150 & 5 & $2244 \pm 25$ & $(47)^{*}$ & straightened \\
\hline
\end{tabular}

*) only one valid measurement

have been chosen for a quantitative comparison of all fibre types as they are independent of the strain measurement. In addition as these values are independent of the measuring length they are easily comparable to literature values. An overview of the obtained results is given in table 1 . For the as-fabricated wire types a significant increase of strength with decreasing diameter has been observed. No significant influence of the testing speed on the strength values of 16 type fibres was detected. For straightened wires the strength is in general lower, and fibres with a diameter of $50 \mu \mathrm{m}$ and $150 \mu \mathrm{m}$ follow the same trend. However, fibres with a diameter of $100 \mu \mathrm{m}$ exhibited similar strength values for both cases. Here the strength of 100 fibres seems to be too low to fit into the scheme. Thinning of the wire from $16 \mu \mathrm{m}$ to $5 \mu \mathrm{m}$ reduced the strength slightly.

Necking was observed for all fibres. The reduction in cross-section of $37 \pm 3 \%$ for the 150 fibres is enhanced for thinner fibres as well as for straightened ones. In figure 2 typical fracture surfaces are shown for 150-th,150,16 and 5-th fibres. All fracture surfaces show a fibrous structure as a result of the knife edge failure of individual grains (see magnified images of fracture surfaces in upper right corners of the sub-figures). The fracture surface of the 150-st,150 and 16 type fibres look very similar showing a crack network including some larger cracks beside the fibrous structure. The 150-st fibre shows some sites near a large crack where the fracture mode locally changes from knife-edge-fracture to brittle cleavage (see white arrows and detail image in figure 2 (a)). Thinning did not change the fracture behaviour significantly and the fracture surface of the 5-th fibres look similar but seems exhibiting a more wrinkled outer surface. 


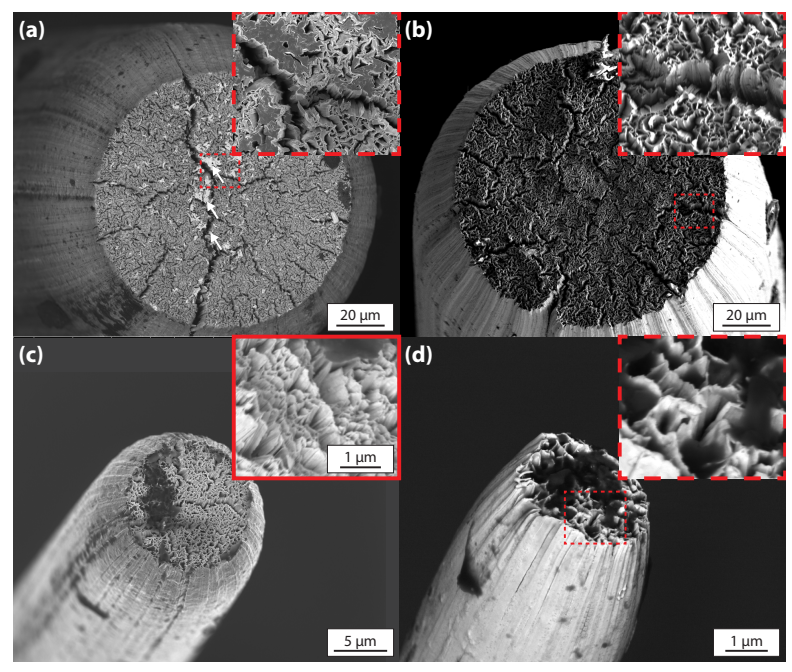

Figure 2. SEM images of a typical fracture surface of (a) 150-st, (b) 150, (c) 16 and (d) 5-th type fibres. In addition the fracture surfaces are shown with a higher magnification in the upper right corner of each sub-figure. Please notice that the detail in (c) shows a different sample. All fibres exhibit necking and a fibrous fracture surface structure caused by the knife edge failure of individual grains. In (a) cleavage fracture near a large crack is indicated by white arrows and shown in a detail image. The blackish area in (d) is caused by non removable carbon containing contamination.

\section{Discussion}

All fibre types show qualitatively similar characteristics in the stress-strain behaviour well known for $\mathrm{W}$ wire $[20,21,8,13]$. Beside the differences in strength, no clear size effect is detectable. A reason for the missing plateau and pronounced load drop prior to failure for the thin fibres in table 1 might be that due to the low maximum strength values in these cases the elastically stored energy in the testing system might lead to an accelerated failure once maximum load is passed.

For the as-fabricated fibres the strength increases as the diameter decreases. As described above a thinner wire requires more drawing steps. In each of these steps the deformation is increased accompanied by dislocation multiplication and an increase of dislocation density. This and the concurrent reduction in grain diameter leads to an increase in strength by strain hardening and grain boundary strengthening during each step. The thinner the wire, the more steps are needed and the higher the strength. On the other hand the reduced strength of the straightened wire can be attributed to a reduction of the dislocation density caused by recovery processes through annealing during the straightening process. This results in a reduced strain hardening capability. For fibres with a diameter of $150 \mu \mathrm{m}$ a similar reduction in strength observed after annealing was also attributed to a reduced dislocation density [8, 13]. There is no increase in strength by diameter reduction from the 16 type fibres to the 5-th type fibres which is as expected as the microstructure is not changed during this process. The slightly reduced strength could be a consequence of variations within the fibres. 


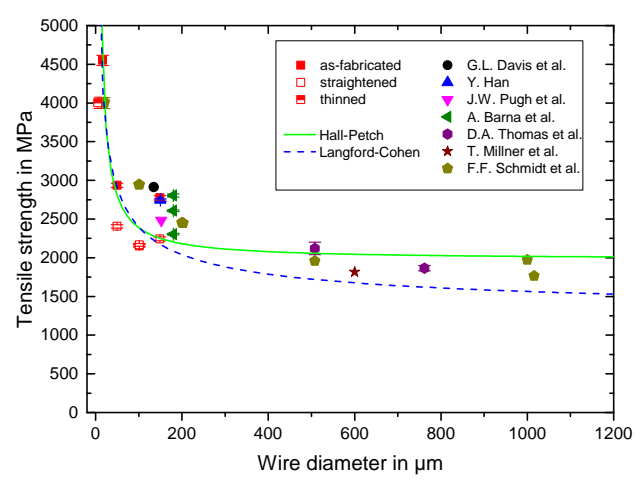

Figure 3. Comparison of strength of drawn tungsten wire with diameter between 5 and $762 \mu \mathrm{m}[8]$.

Also the different surface conditions of as-fabricated and electropolished material may have an influence. However, the restricted number of tests has to be taken into account and further tests are planned for a better understanding.

A reason for the similar strength values for the as-fabricated and straightened fibres with a diameter of $100 \mu \mathrm{m}$ could be the fabrication history. As mentioned above, intermediate annealing steps are needed during the drawing process. The exact order of drawing and annealing steps has significant influence on the number of mobile dislocations and thus the wires work hardening ability and hence on its ultimate strength [17]. An annealing step in the final state of the production leads to a reduced dislocation density and thus a reduced ultimate strength. This seems to be the case for the asfabricated wire with a diameter of $100 \mu \mathrm{m}$. Due to a already low dislocation density the additional annealing during straightening only has a minor effect on the dislocation density and thus on the strength. Microstructural investigations are planned to compare the amount of dislocations for the two different wire types as in this case both should be similar. This theory is backed by the fact that the as-fabricated 100 type fibres only show a minor work hardening during the tension tests.

In figure 3 the results for ultimate strength are summarized and compared to literature values. As discussed straightening as well as intermediate annealing steps can have a significant influence on the observed mechanical behaviour and more precisely on the strength values. This variation has to be taken into account if comparing results of different tension tests. The difference between the here measured as-fabricated and straightened wire of $\Delta \sigma_{\mathrm{u}}=331 \pm 28 \mathrm{MPa}$ can be taken as a first estimation. However, wherever possible the exact fabrication history should be considered.

The fracture behaviour with pronounced necking and the typical fibrous fracture of individual grains is typical for drawn $\mathrm{W}$ wire $[22,8,13]$. The observed crack network for the 16,150 and 150-st type fibres does probably originate in surface grooves caused by the fabrication process and now acting as crack starting point $[13,14]$. The reduction in cross-sectional area as a measure for ductility is similar 
to literature values for the 150 type fibre [15] and rises for very thin fibres. This corresponds with observations for wire with a diameter of $64 \mu \mathrm{m}$ where the reduction in area was $45 \%$ [20]. With respect to the restricted sample number a clear trend for 5-th fibres can not be given. The reduced area in the 150-st although only measured for one sample corresponds well with the results observed for annealed wire [13]. In these wires a reduced dislocation density results in an enhanced mean free path and thus an easier movement of the dislocations which can be beneficial for necking. Whether this can also be the explanation for the enhanced ductility in thin fibres can be proven by a comparison of the respective microstructures and especially dislocation densities. More tests combined with a detailed microscopical analysis are planned to enhance the database and understand the underlying mechanisms.

The cleavage fracture detected in 150-st fibres has been also observed for annealed wire in [13] and was attributed to a lower present stress. The large crack observed near the cleavage area could have had a similar effect of stress reduction. The wrinkling of the 5-th fibres might be a consequence of electro polishing, leading to a reduction of surface grooves. The absence of crack initiation points could promote wrinkling rather than the formation of cracks However the very small size of these samples might also play a role and need further investigation. As a first step tests off drawn wires with a surface finish by electro polishing are planned .

The metallurgical reasons for the observed results will be discussed in the following. The characteristic microstructure of drawn tungsten wire shows a large amount of grain boundaries $[23,24,25]$. This supports the assumption that grain boundaries act as obstacles for dislocation motion and therefore increase the strength (and ductility) of tungsten wire - a process known as grain boundary hardening. In order to evaluate this assumption, the mechanical properties obtained in the uni-axial tensile tests were analysed. The tensile strength, which is defined as the maximum stress observed in a tensile test, was used for evaluating the strengthening mechanisms of drawn tungsten wire. It is common to plot a mechanical parameter such as the tensile strength against the (mean) grain size of a material in order to study its strengthening behaviour. As a quantitative and precisely to measure parameter with direct relationship to the transversal grain size is the wire diameter [26, 12] was chosen in this case. This, of course can only give a first estimation as the role of dislocations as well as the role of the fabrication history is neglected.

Two different models were used to describe the grain boundary hardening mechanisms. The model by Hall [27] and Petch [28] (Hall-Petch), describing the relation between a mechanical parameter and grain diameter $d$ using the constants $\sigma_{0}$ and $k$. The related formula with the tensile strength as mechanical parameter and adopted for the wire diameter instead of the grain size is the following:

$$
\sigma_{\mathrm{u}, \mathrm{HP}}(d)=\sigma_{0, \mathrm{HP}}+\frac{k_{\mathrm{HP}}}{\sqrt{d}}
$$

A modification of equation (1) was proposed by Langford and Cohen [29] (LangfordCohen). The difference between the models manifests in a different exponent of the 
Tensile behaviour of drawn tungsten wire

Table 2. Parameters for Hall\&Petch and Langford\&Cohen equations.

\begin{tabular}{lcccc}
\hline Model & Exponent $n$ & $\sigma_{0, i}$ in $\mathrm{MPa}$ & $k_{i}$ in $\mathrm{MPa} \mathrm{m}^{\mathrm{n}}$ & $i$ \\
\hline Hall-Petch & 0.5 & 1179 & 12.16 & $\mathrm{HP}$ \\
Langford-Cohen & 1 & 1977 & 0.04 & LC \\
\hline
\end{tabular}

Table 3. Strength of W single crystals.

\begin{tabular}{ccc}
\hline Reference & Geometry & Tensile strength $\sigma_{\mathrm{y}}$ in $\mathrm{MPa}$ \\
\hline$[33]$ & rod & 750 \\
{$[34]$} & rod & $860 \pm 50$ \\
\hline
\end{tabular}

grain size or the wire diameter in this case:

$$
\sigma_{\mathrm{u}, \mathrm{LC}}(d)=\sigma_{0, \mathrm{LC}}+\frac{k_{\mathrm{LC}}}{d}
$$

Only results of wire in the as-produced condition, tested with a cross-head speed of $5 \mathrm{\mu m} \mathrm{s}^{-1}$ are used for the model description to avoid the influence of the testing condition, of wires with various diameter are planned to improve the database. Nevertheless, by comparing the positions of the data points in figure 3 and the curve characteristics, one 
can clearly state that the high strength of drawn tungsten wire is at least partially due to grain boundary hardening mechanisms.

\section{Summary and consequences for $\mathrm{W}_{\mathrm{f}} / \mathrm{W}$ composites}

Tensile tests on tungsten wires with different diameter reveal a strong relationship between strength and diameter as well as fabrication history. The most important findings are:

- Reducing the diameter by drawing generally increases the strength up to $4500 \mathrm{MPa}$ for wire with a diameter of $16 \mu \mathrm{m}$.

- A grain boundary hardening mechanism contributes to the high strength of drawn tungsten wire.

- The tensile behaviour of $\mathrm{W}$ wire is not significantly changed by the reduction of the diameter by drawing.

To investigate the relationship between diameter, grain size and dislocation detailed microstructural investigations are ongoing. Tensile tests and EBSD measurements of wires with larger diameter will allow expanding the database and thus a better description of the deformation behaviour and a better correlation with the hardening models.

It was shown that wire with a diameter of $16 \mu \mathrm{m}$ has a significantly increased strength compared to $150 \mu \mathrm{m}$ wire which is at the moment mainly used wire in $\mathrm{W}_{\mathrm{f}} / \mathrm{W}$. By using the $16 \mu \mathrm{m}$ wire in $\mathrm{W}_{\mathrm{f}} / \mathrm{W}$ the bridging effectiveness could be significantly increased due to the significantly increased strength. However, the handling of this very thin wire during the manufacturing process will be challenging. It is planned to develop multifilament yarns consisting of $16 \mu \mathrm{m}$ thin $\mathrm{W}$ wire filaments to be able to utilize the good properties and ensure an easy handling. In addition the results showed that the exact quantitative strength values are very much determined by the fabrication process e.g. the performed intermediate annealing steps. It is therefore essential to be aware of the exact fabrication histories of the wires to be used in $\mathrm{W}_{\mathrm{f}} / \mathrm{W}$. Independent of the wire diameter all fibre types show qualitatively similar characteristics in the stress-strain behaviour showing the same deformation characteristics and only a variation in strength. Also thinning does not seem to alter the mechanisms underlying the deformation behaviour significantly. The absence of a size effect opens the possibility to use thin wires for studies requiring small specimen sizes and extrapolate the results to thick wire.

\section{Acknowledgements}

The authors want to thank G. Matern, M. Balden and S. Elgeti for their assistance in microscopy and A. Manhard for his support in electrochemical thinning. This work has been carried out within the framework of the EUROfusion Consortium and has received funding from the Euratom research and training programme 2014-2018 under 
grant agreement No 633053. The views and opinions expressed herein do not necessarily reflect those of the European Commission.

\section{Appendix A. Error analysis ultimate strength}

In order to evaluate the accuracy of the strength values of the tungsten wires, an error analysis was performed. Therefore, the equations for the relative error limits resulting from the Gaussian error propagation were utilized. This yields the following formula for the tensile strength $\sigma_{\mathrm{u}}$ of a drawn tungsten wire with an initial diameter $d$ :

$$
\Delta \sigma_{\mathrm{u}}=\sigma_{\mathrm{u}} \cdot\left(\frac{\Delta F}{F}+\frac{2 \Delta d}{d}\right)
$$

The relative error of the force $F, \frac{\Delta F}{F}$, is specified to be 0,025 by the manufacturer of the

utilized load cells. The second term in equation (A.1) arises from the contribution of the cross-sectional area of the tested wires, which was approximated to be a circle with a diameter $d$. The error of the wire diameter was calculated from direct measurements for the wire types 150 and 100 (see table 1). The error for the remaining wire types were then approximated by a parabolic relation between the diameter provided by the wire manufacturer and its scatter.

\section{References}

[1] Coenen J W, Antusch S, Aumann M, Biel W, Du J, Engels J, Heuer S, Houben A, Hoeschen T, Jasper B, Koch F, Linke J, Litnovsky A, Mao Y, Neu R, Pintsuk G, Riesch J, Rasinski M, Reiser J, Rieth M, Terra A, Unterberg B, Weber T, Wegener T, You J H and Linsmeier Ch 2016 Physica Scripta T167 014002-

[2] Lassner E and Schubert W D 1999 Tungsten - Properties, Chemistry, Technology of the Element, Alloys, and Chemical Compounds (Kluwer Academic / Plenum Publishers)

[3] Yih S and Wang C 1979 Tungsten: Source, Metallurgy, Properties, and Applications (Springer Science+Business Media New York)

[4] Barabash V, Federici G, Rödig M, Snead L and Wu C 2000 Journal of Nuclear Materials 283-287 $138-146$

[5] Riesch J, Höschen T, Linsmeier Ch, Wurster S and You J H 2014 Physica Scripta T159 014031-

[6] Riesch J, Coenen J, Gietl H, Höschen T, Mao Y, Linsmeier Ch and Neu R 2016 Tungsten fibrereinforced tungsten composite - development of a new high performance material Proceedings of the 17th European Conference on Composite Materials ISBN 978-3-00-053387-7

[7] Linsmeier Ch, Rieth M, Aktaa J, Chikada T, Hoffmann A, Hoffmann J, Houben A, Kurishita H, Jin X, Li M, Litnovsky A, Matsuo S, Müller A, Nikolic V, Palacios T, Pippan R, Qu D, Reiser J, Riesch J, Shikama T, Stieglitz R, Weber T, Wurster S, You J H and Zhou Z 2016 Nuclear Fusion Accepted

[8] Riesch J, Han Y, Almanstötter J, Coenen J W, Höschen T, Jasper B, Zhao P, Linsmeier Ch and Neu R 2016 Physica Scripta T167 014006-

[9] Gietl H, Riesch J, Coenen J, Höschen T, Linsmeier Ch and Neu R 2017 Fusion Engineering and Design - http://dx.doi.org/10.1016/j.fusengdes.2017.02.054

[10] Riesch J, Buffiere J Y, Höschen T, di Michiel M, Scheel M, Linsmeier Ch and You J H 2013 Acta Materialia 61 7060-7071 
[11] Riesch J, Buffiere J Y, Höschen T, Scheel M, Linsmeier Ch and You J H 2017 Composites Part A: Applied Science and Manufacturing Submitted

[12] Schade P 2010 International Journal of Refractory Metals and Hard Materials 28 648-660

[13] Zhao P, Riesch J, Höschen T, Almannstötter J, Balden M, Coenen J, Himml R, Pantleon W, von Toussaint U and Neu R 2017 International Journal of Refractory Metals and Hard Materials Submitted

[14] Terentyev D, Riesch J, Lebediev S, Bakaeva A and Coenen J 2017 International Journal of Refractory Metals and Hard Materials 66 127-134 ISSN 0263-4368

[15] Riesch J, Almanstötter J, Coenen J W, Fuhr M, Gietl H, Han Y, Höschen T, Linsmeier Ch, Travitzky N, Zhao P and Neu R 2016 IOP Conf. Series: Materials Science and Engineering 139

[16] Coolidge W D 1913 Tungsten and method of making the same for use as filaments of incandescent electric lamps and for other purposes uS patent 1,082,933

[17] Mullendore J 1989 The Metallurgy of Doped/Non-Sag Tungsten (Elsevier) chap The technology of doped-tungsten wire manufacturing, pp 61-82

[18] Schultz H 1964 Acta Metallurgica 12 649-664

[19] Manhard A, von Toussaint U, Balden M, Elgeti S, Schwarz-Selinger T, Gao L, Kapser S, Pociski T, Grzonka J, Gloc M and Ciupiski 2017 Nuclear Materials and Energy http://dx.doi.org/10.1016/j.nme.2016.10.014

[20] Lee D 1975 Metallurgical Transactions A 6A 2083-2088

[21] Harris B and Ellison E 1966 Trans. Amer. Soc. Metals 59 744-754

[22] Leber J, Tavernelli J, White D and Hehemann R 1976 Journal of Less-Common Metals 48 119-133

[23] Meieran E and Thomas D 1965 Transactions of the Metallurgical Society of AIME 233 937-943

[24] Barna A, Gaal I, Geszti-Herkner O, Radnoczi G and Uray L 1978 High Temperatures - High Pressures 10 197-205

[25] Walter J L and Koch E F 1991 Journal of Materials Science 26 505-509 ISSN 1573-4803

[26] Ripoll M R 2009 Drawing of Tungsten Wires: Microstructure, Mechanical Properties and Longitudinal Cracks Ph.D. thesis Institut für Werkstoffkunde I: Institut für Zuverlässigkeit von Bauteilen und Systemen Universität Karlsruhe

[27] Hall E O 1951 Proceedings of the Physical Society. Section B 64 747-

[28] Petch N 1953 Journal of Iron and Steel Research International $\mathbf{1 7 4}$

[29] Langford G and Cohen M 1970 Metallurgical and Materials Transactions 1 1478-1480

[30] Hirth J P 1972 Metallurgical Transactions 3 3047-3067

[31] Li Y, Bushby A J and Dunstan D J 2016 Proceedings of the Royal Society A: Mathematical, Physical and Engineering Science $\mathbf{4 7 2}$ -

[32] Gottstein G 2007 Physikalische Grundlagen der Materialkunde (Springer Verlag Berlin Heidelberg)

[33] URL https://www . americanelements . com/tungsten-single-crystal-7440-33-7

[34] Garfinkle M 1965 Room-temperature tensile behaviour of $<100>$ oriented tungsten single crystals with rhenium in dilute solid solution NASA Technical Note 20060516196 Lewis Research Center

[35] Lesuer D R, Syn C K and Sherby O D 2010 Journal of Materials Science 45 4489-4894 